\title{
Necessity of Reorganization of Mental Health Welfare Act to Decrease in Social Anxiety Related to Patients with Schizophrenia and to Ensure Proper Human Rights for Mentally Ill Persons
}

\author{
Hoseon Lee ${ }^{1}$, Joonho Choi ${ }^{\bowtie}$, Myung Soo Lee ${ }^{2}$, Jong-Woo Paik ${ }^{3}$, and Jun Soo Kwon ${ }^{4}$ \\ ${ }^{1}$ Department of Psychiatry, Hanyang University Guri Hospital, Guri, Republic of Korea \\ ${ }^{2}$ Yonsei Life Psychiatric Clinic, Seoul, Republic of Korea \\ ${ }^{3}$ Department of Psychiatry, Kyunghee University College of Medicine, Seoul, Republic of Korea \\ ${ }^{4}$ Department of Psychiatry, Seoul National University College of Medicine, Seoul, Republic of Korea
}

The Korean Neuropsychiatric Association (Chairman: Jun Soo Kwon) is deeply regretful of the recent accidents caused by mental illness patients, and it is necessary to construct a system for optimal treatment of mental illness. We would like to assert that this is a way to ensure genuine human rights and resolve social uneasiness. We call for reorganization of the Mental Health Welfare Act (Act on the Improvement of Mental Health and the Support for Welfare Services for Mental Patients).

We need to take special measures to improve the current mental health system that makes mental illness patients criminals. The Mental Health Welfare Act, which was amended in 2015 and enacted on May 30, 2016, has complicated involuntary hospitalization procedure includes Hospitalization by legal guardian (referring to two or more persons with priority according to the order of priority), a two-psychiatrist's diagnosis at different medical institutions and a screening Committee for Examination as to Legitimacy of admission and committee for admission and a mental health welfare review committee, But there is no countermeasure against the mentally ill who are neglected in the blind spot.

Making the admission procedure complicated cannot be a necessary and sufficient condition to guarantee the human rights of the patient. It is important to be able to run tight care and community management programs to maintain the mental health of those who are discharged repeatedly and repeat recurrence. It should not be left untreated mental illness patients in the community because the risk of mentally ill patients' self-

\footnotetext{
$\triangle$ Correspondence: Joonho Choi, MD, PhD

Department of Psychiatry, Hanyang University Guri Hospital, 153 Gyeongchun-ro, Guri 11923, Republic of Korea

Tel: +82-31-560-2277, Fax: +82-31-554-2599, E-mail: jchoi@hanyang.ac.kr

(c) This is an Open Access article distributed under the terms of the Creative Commons Attribution Non-Commercial License (http://creativecommons.org/licenses/bync/4.0) which permits unrestricted non-commercial use, distribution, and reproduction in any medium, provided the original work is properly cited.
}

harm or threat to others is not clear. Various types of intervention strategies, such as involuntary outpatient commitment, should be developed as in developed countries.

In the medical situation, psychiatrist often experience situations in which patients are unable to treat despite of the need for treatment. If the risk of self-harm or threat to others is ambiguous, no treatment can be done without patient consent. Government and public systems have no mechanism to help these patients, and the burden of care is passed on entirely to the family. It is a sad reality that we have to tell the guardian that they have to wait for the danger of being struck.

Even if it is for the guarantee of human rights, the two-psychiatrist's diagnosis' and 'Committee for Examination as to Legitimacy of admission' system contains contradictory contents. We must assess the admission suitability at the time of admission. It is not reasonable that the time of decision is at 30 days after admission. It is also not appropriate to have an assessment of the diagnosis of two psychiatrists. In order to evaluate the procedural legitimacy, it is necessary to establish an infrastructure and an operating system that can assess the adequacy of the entire visa at the beginning of the hospital as in developed countries. The Korean Neuropsychiatric Association has repeatedly proposed the need for a judicial or quasi-judicial admission system. Nevertheless, the Ministry of Health and Welfare only rely on written examination due to lack of manpower and budget. And they are also pushing to discharge patients who need more treatment under the guise of the decision of the committee. It is only for the ranking in the OECD nations but not for the untreated patients.

We know that we are making policy efforts to place additional workforce in the local mental health welfare center, but the proverb "to pee in the freezing" is just right. Patients with well-recovered schizophrenia suffer from indictments of accidents involving patients with the same diagnosis and 
are anxious that they can do that.

The Ministry of Health and Welfare should no longer allow sick patients to be stigmatized as potential criminals and turned away from society. In order to guarantee the human rights, the system of an involuntary admission should be completely redeveloped and special measures should be taken to provide tight support without blind spots. It is no longer possible for patients and families to carry these heavy burdens. It is important for the human rights of the patient and the fam- ily to improve the system to ensure optimal treatment so that the patient can recover from illness and enjoy a healthy life. People who commit crimes should be punished, but they must also be held accountable for neglecting preventable crime.

The Korean Neuropsychiatric Association will make every effort to amend the law and improve the system in the nearest future, and will continue to inform and communicate to the government, the National Assembly, the parties, the family groups and the people. 